\title{
Selection of optimal bands for developing multispectral system for inspecting apples for defects
}

I. Baek, C. Eggleton, S. A. Gadsden, M. S. Kim

I. Baek, C. Eggleton, S. A. Gadsden, M. S. Kim, "Selection of optimal bands for developing multispectral system for inspecting apples for defects," Proc. SPIE 11016, Sensing for Agriculture and Food Quality and Safety XI, 110160F (30 April 2019); doi: 10.1117/12.2520469

EDIE Event: SPIE Defense + Commercial Sensing, 2019, Baltimore, Maryland, United States 


\title{
Selection of optimal bands for developing multispectral system for inspecting apples for defects
}

\author{
I. Baek ${ }^{\mathrm{a}, \mathrm{b}}$, C. Eggleton ${ }^{\mathrm{b}}$, S. A. Gadsden ${ }^{\mathrm{c}}$, M. S. Kim ${ }^{\mathrm{a}, *}$ \\ ${ }^{a}$ Environmental Microbial and Food Safety Laboratory, Agricultural Research Service, U.S. Dept. of \\ Agriculture, Powder Mill Rd. Bldg. 303, BARC-East, Beltsville, MD USA 20705; ${ }^{\mathrm{b}}$ Dept. of \\ Mechanical Engineering, University of Maryland Baltimore County, 1000 Hilltop Circle, Baltimore, \\ MD USA 21250; ${ }^{c}$ College of Engineering and Physical Sciences, University of Guelph, 50 Stone \\ Rd East, Guelph, Ontario, Canada, N1G 2W1
}

\begin{abstract}
Hyperspectral image technology is a powerful tool, but oftentimes the data dimension of hyperspectral images must be reduced for practical purposes, depending on the target and environment. For detecting defects on a variety of apple cultivars, this study used hyperspectral data spanning the visible $(400 \mathrm{~nm})$ to near-infrared $(1000 \mathrm{~nm})$. This paper presents the preliminary results from the selection of optimal spectral bands within that region, using a sequential feature selection method. The selected bands are used for multispectral detection of apple defects by a classification model developed using support vector machine (SVM). As a result, five optimal wavelengths were selected as key features. When using optimal wavelengths, the accuracy of the SVM and SVM with RBF kernel achieved accuracies over $90 \%$ for both the calibration and validation data set. However, the results of SVM with RBF kernel $(>80 \%)$ based on image was more robust than SVM model $(>50 \%)$. Moreover, SVM with RBF model classified between bruise and sound regions as well specular. The result from this study showed the feasibility of developing a rapid multispectral imaging system based on key wavelengths.
\end{abstract}

Keywords: apple, bruise, SVM, SFS, image

\section{INTRODUCTION}

Bruises on apples usually result from external forces during harvest, transportation or on a packaging line ${ }^{1,2}$. These are difficult to visually detect in a short time period since the bruised regions develop beneath the apple skin and gradually change the color of skin. Bruise damage can be lead nutrient loss, premature decay and a reduction in the economic price of the apple ${ }^{3}$. Thus, bruised apples should be separated before going to the market place. Many proposed methods for detecting bruised regions of apples have been published using hyperspectral imaging systems (HIS). Janos et al. (2016) ${ }^{1}$ describeed the detection system for Jonagold apple bruises using short wave infrared hyperspectral imaging. Bruised regions on Golden Delicious apples were detected by multiple wavebands between 400 and $1000 \mathrm{~nm}{ }^{2}$, and the AdaBoost algorithm were developed for detecting bruises on Fuji apples ${ }^{4}$ through visible and near infrared wavelengths. These studies paid attention to detection of bruises on one selected apple cultivar with a few wavebands or several cultivars with ten or more wavebands. This should be enough to sort apples on commercial line. However, on the consumer side, the inspection method for bruised apples should detect bruises on various cultivar apples by a simple and fast method. For example, it is possible for a consumer to inspect apples onsite using a device before make a purchase. The consumer benefits from high-quality apples. Moreover, the seller can provide reliability to the consumer.

The application of the feature selection and classification method has become efficient in distinguishing bruises and sound regions of apples. The sequential forward selection (SFS) method was used for selecting less than five optimum wavebands. The SFS method is one of the feature selection methods, which select a subset of the existing features without a transformation. Therefore, this method can reduce the processing time and cost required to identify features. For example, studies related to hyperspectral imaging have $b$ focused on finding optimal wavelengths for detecting or evaluating surface features. Because hyperspectral imaging data consist of large file sizes that take a long time to obtain when the full spectrum of wavelengths is considered., This is not suitable for an online-system. It is more efficient to select optimal wavebands for feature selection. SFS starts with an empty subset, and each interaction adds to the subset through the evaluation score. In this investigation, a support vector machine (SVM) was applied for detecting bruised

Sensing for Agriculture and Food Quality and Safety XI, edited by Moon S. Kim, Bryan A. Chin, Byoung-Kwan Cho, Proc. of SPIE Vol. 11016, 110160F - (C) 2019 SPIE · CCC code: 0277-786X/19/\$18 · doi: 10.1117/12.2520469 
damage on apple surfaces as a classifier. SVM has been used widely in agricultural fields since it is generalizable and robust. In this paper, a detection method using SVM with a few optimum wavebands is presented.

\section{MATERIALS AND METHODS}

\subsection{Apple and Bruising procedure}

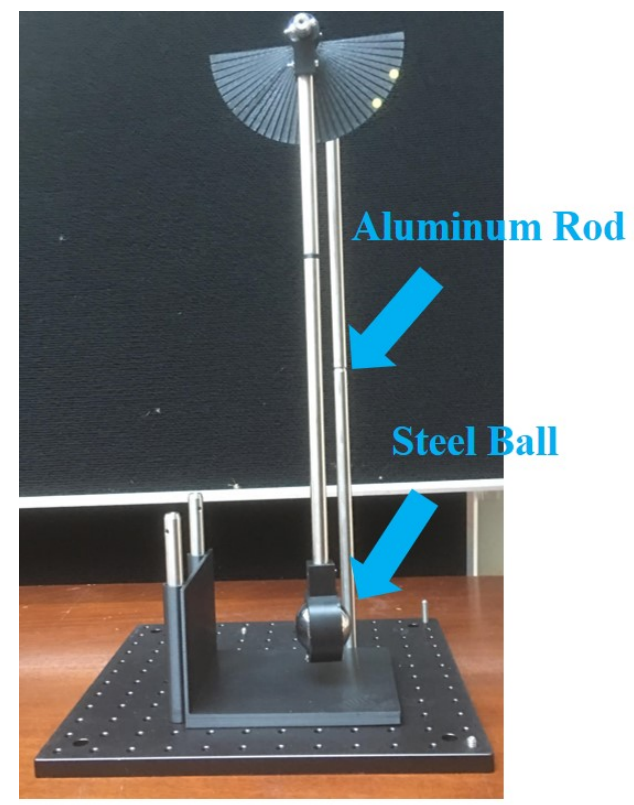

(a)

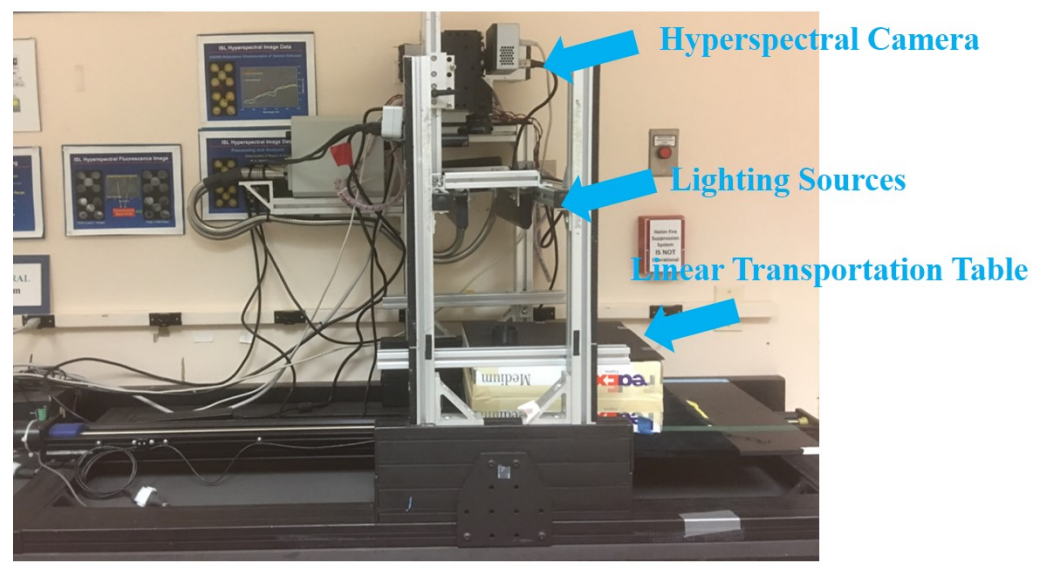

(b)

Figure 1. Photos of (a) an equipment for developing bruises on apple and (b) the hyperspectral imaging system.

Three cultivars of apples (Golden Delicious, Red Delicious and Fuji) were purchased from a local market in Beltsville (United State). 10 apples of each cultivar were selected with various size, color, shape and nonvisual defect. Three different levels $(1.11,0.66$ and $0.33 \mathrm{~J})$ of impact energy were utilized to induce bruises on apple. The impact energy $\mathrm{E}$ (in J) was calculated by Eq. $1^{5}$. 


$$
\mathrm{E}=m_{b} g h+m_{r} g \frac{h}{2}
$$

Where $m_{b}$ and $m_{r}$ are the mass of the steel ball and an aluminum rod $(\mathrm{kg}), \mathrm{g}$ is the gravity of acceleration $\left(\mathrm{m} / \mathrm{s}^{2}\right)$ and $\mathrm{h}$ is the height $(\mathrm{m})$ of the steel ball from ground.

Fig. 1 shows the design of the equipment for inducing bruises on apples. This equipment consisted of steel ball and aluminum rod. The $357 \mathrm{~g}$ steel ball was mounted onto an aluminum rod which is $45 \mathrm{~cm}$ in length and $433 \mathrm{~g}$ in weight. The other end of the steel road was mounted onto a pivot. Thus, steel ball would act as a simple pendulum device. The apples were placed at sample holder while the steel ball and rod fall onto the apple's equator. The bruises on apple were developed by rotating apple with the equator direction so that the three bruises by different impact energy were induced on one sample. Hyperspectral images at 6 and $24 \mathrm{~h}$ after bruising were obtained. There were 180 hyperspectral images were acquired ( 3 cultivars $\mathrm{x} 3$ different impact energy x 2 different time x 10 samples).

\subsection{Acquisition of hyperspectral images}

As shown in the fig. 1 (b), the hyperspectral camera include an electron-multiplying charge-coupled device camera (EMCCD : Luca R DL-604M, 14-bit, Andor Techonology, South Windsor, CT, USA), a C-mount objective lens and visible and near infrared imaging spectrograph. The apple bruises were placed toward the objective lens on a programmable linear stage with a stepping motor. The HIS was designed to cover visible (VIS) to near-infrared (NIR) wavelengths for relating reflectance measurements. The $150 \mathrm{~W}$ halogen lamps were used as a light sources and uniformly illuminated the sample via fiber optic. In this investigation, the hyperspectral images were calibrated by Eq. 2, called as flat-field correlation method since raw hyperspectral images contained noise and artifacts related to measurement environments and imperfections of each component (e.g., source, lens, filter, spectrograph, and camera) ${ }^{6,7}$.

$$
\mathrm{I}_{\mathrm{R}}=\frac{I_{r}-I_{d}}{I_{w}-I_{d}}
$$

Where $I_{R}$ is raw hyperspectral image, $I_{d}$ is dark current image and $I_{w}$ is reference image.

\subsection{Analyzing algorithm}

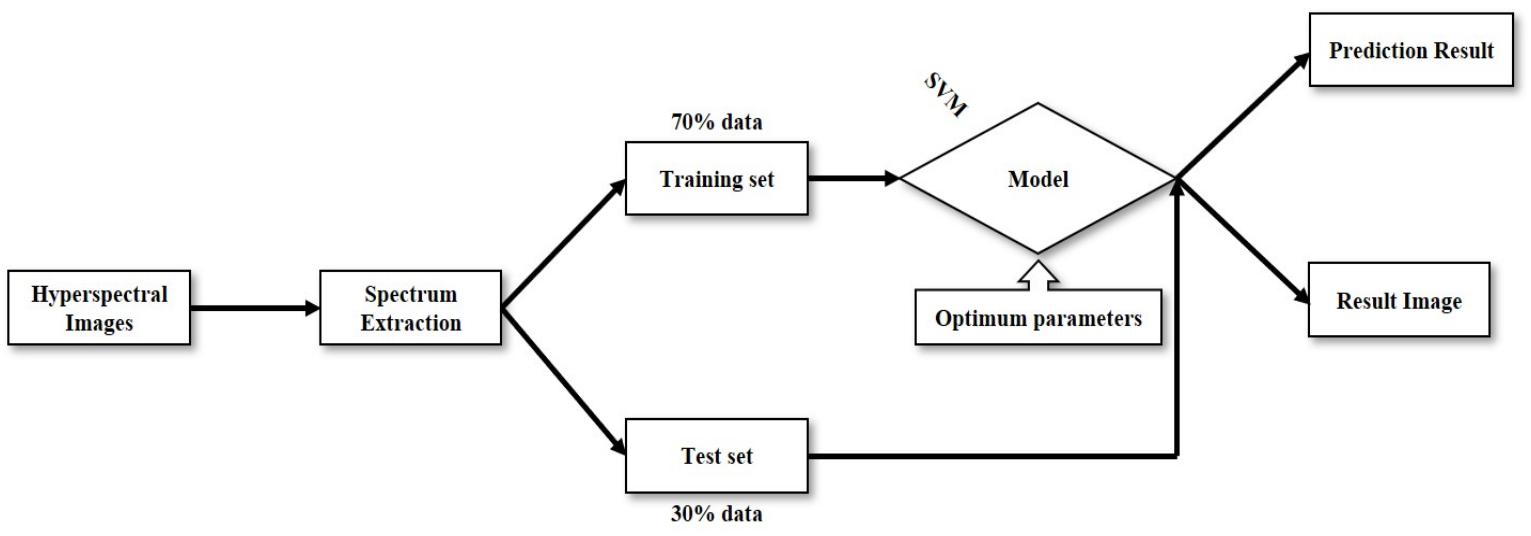

Figure 2. Key procedure steps used for the classification of bruises on apple.

The region of interest (ROI) was conducted to extract the spectrum data from the hyperspectral image by manual selection. It was difficult to select bruise region by one band image. Thus, principle component images were used for selecting bruise regions, precisely. For construction of the SVM model, the entire spectrum data obtained from three different bruise levels and three cultivar apples at 6 and 24 hours. The data were split into two subsets (calibration and validation data sets). Calibration and validation sets contained $70 \%$ and $30 \%$ of the total data, respectively. In this study, 
SVM and SVM with Gaussian radial basis function (RBF) were used for classifying between sound and bruised regions on apple. The parameter of cost function (c) and gamma $(\gamma)$ were optimize by a grid search method. The developed SVM model was applied to hyperspectral images, and then the bruised region on apple was visualized with red color.

\section{RESULT AND DISCUSSION}

\subsection{Mean spectrum and optimum wavebands}

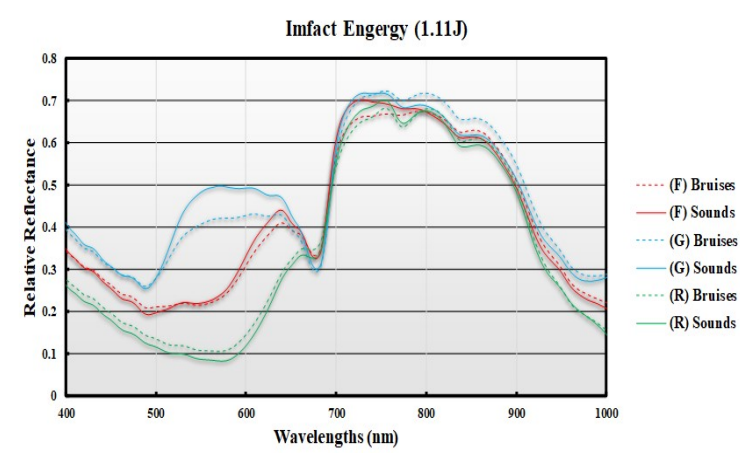

(a)

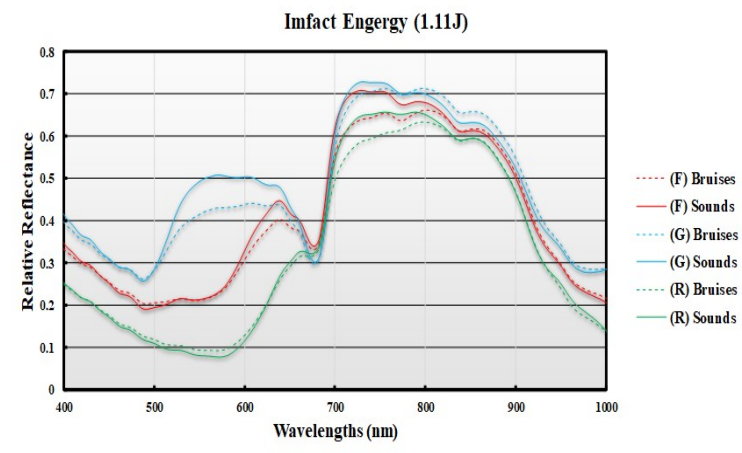

(b)

Figure 3. The average spectrum of bruise and sound of apples in 6 (a) and 24 hours (b).

Figure 3 presents the mean spectra of three cultivar apples after developing bruises for 6 and 24 hours. The characteristic browning symptom was shown between 550 and $650 \mathrm{~nm}^{2}$. Moreover, the spectrum for each cultivar showed valleys near $680 \mathrm{~nm}$, that is mainly associated with the carotenoids and chlorophyll pigments in the apple ${ }^{8}$. Figure 4 indicates the accuracy for the number of wavebands used, from one to six. As a result, two methods using five wavebands obtained a high accuracy with $>90 \%$. However, SVM with RBF attained slightly higher accuracy than SVM model. Crucial to the process of choosing the optimal number of wavebands is the trade-off between performance accuracy and the number of wavebands used in the analysis. Increasing the number of wavebands leads to longer data processing time owing to the increased volume of the image data. Thus, the number of wavebands should be carefully considered. In this study, five wavelengths were chosen as optimal wavebands for further imaging processing, as there was no significant enhancement of the accuracy of the method when adding more wavebands. The summary of the selected optimum wavebands was presented in table 1 . The classification of a bruised and a sound peel was mainly determined in NIR region, since the three cultivars of apple have different colors. The NIR region has the advantage of the free-color influences. In addition to the free-color influences, when the bruise was developed, the cell in the apple tissue was damaged and the intercellular air spaces were decreased, which led to different water content between bruises and sound regions ${ }^{9}$. Hence, NIR from 750 to $1000 \mathrm{~nm}$, which associated with the water absorption bands ${ }^{9}$, presented a predominant wavebands for classifying bruises.

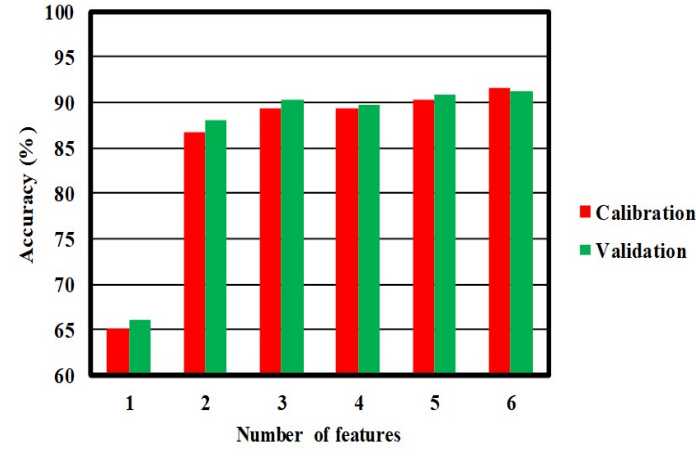

(a)

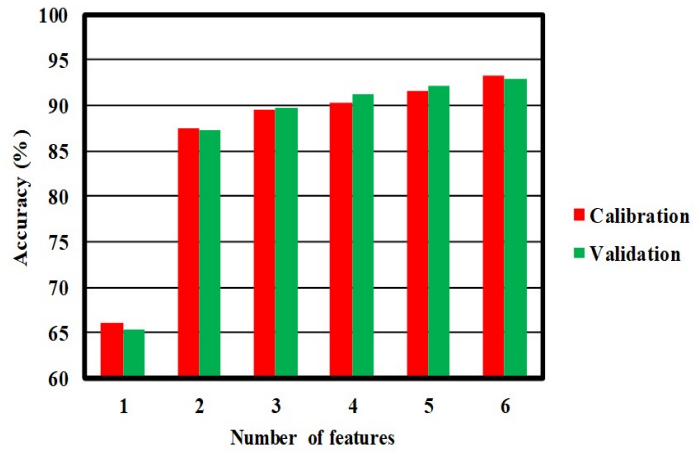

(b)

Figure 4. performance comparing of number of features; (a) SVM and (b) SVM with radial basis function (RBF) kernel. 
Table 1. Selected five most important wavebands by the sequential forward selection method.

\begin{tabular}{cc}
\hline Classifier & Selected wavebands (nm) \\
\hline SVM (linear) & $453,731,740,812,874$ \\
SVM with RBF kernel & $535,726,817,822,865$ \\
\hline
\end{tabular}

\subsection{Image based classification}

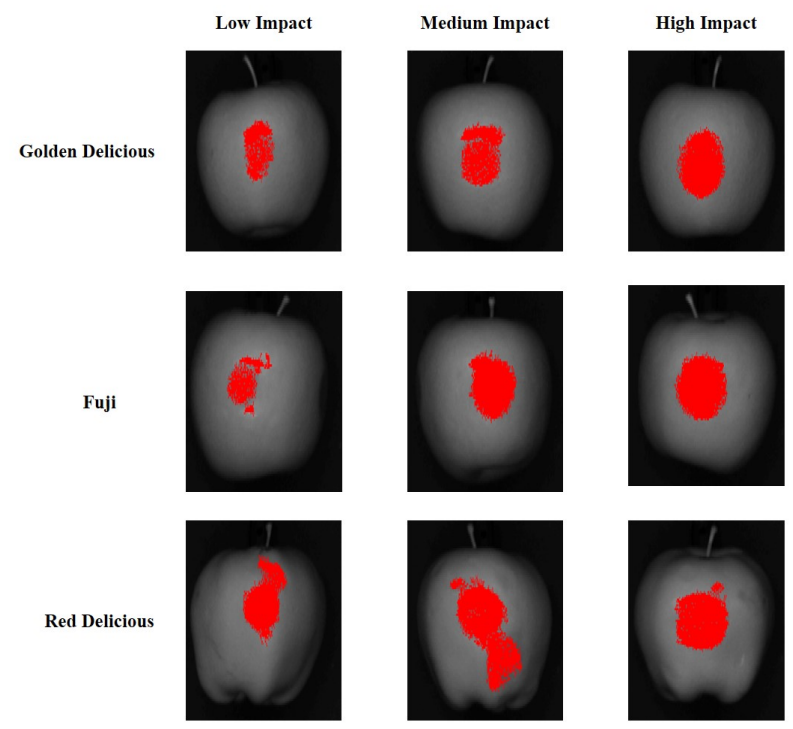

(a)

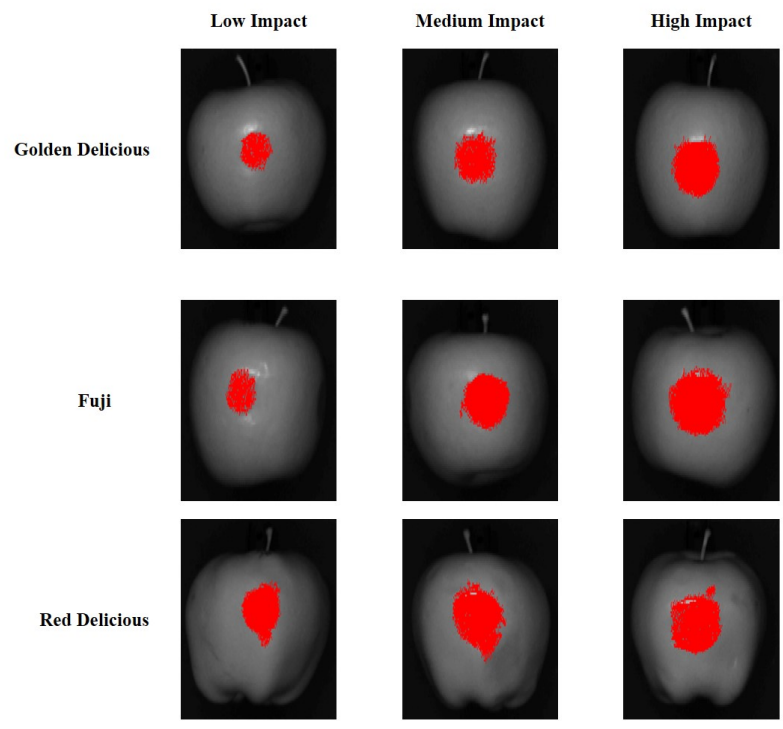

(b)

Figure 5. Visualization of result image with (a) SVM and (b) SVM RBF by using five wavebands after developing bruises for 24 hours. 
Pixel intensity of apples that are less than or equal to the threshold values (0) were classified as bruises on apples and bruise regions were represented in red in the resulting image. The resulting image of SVM with RBF kernel clearly show the bruise regions on the apples. However, the resulting image of SVM has a misclassification on specular regions. In applying the SVM model to online inspection, the detection system needs to prevent misclassification of specular regions by using polarizing filters. Alternatively, specular regions can be removed by additional image processing. The resulting images of three cultivar apples at three levels of bruising were presented. Low impact energy developed smaller bruises than the higher energy levels. The results suggested that SVM-RBF kernel can potentially deal with online processing for detecting bruised regions in market place. Table 2 and 3 show the accuracy of bruise detection based on image analysis. SVM has low accuracy for bruises on fuji with low impact energy and bruises on red delicious at 6 hour. However, this model worked well at 24h. In contrast SVM, SVM with RBF kernel model has high accuracy as shown in table 3. The detection accuracy for this model for $6 \mathrm{~h}$ bruise on red delicious is a little lower than the other two cultivars. Overall, this accuracy indicated that the SVM with RBF kernel model is more robust than the SVM model.

Table 2. Result of classification based on image using SVM.

\begin{tabular}{|c|c|c|c|c|}
\hline Cultivar & Time & Low (\%) & Medium (\%) & High (\%) \\
\hline \multirow{2}{*}{ Fuji } & 6 & 40 & 90 & 100 \\
\hline & 24 & 90 & 100 & 100 \\
\hline \multirow{2}{*}{ Golden delicious } & 6 & 100 & 100 & 100 \\
\hline & 24 & 100 & 100 & 100 \\
\hline \multirow{2}{*}{ Red delicious } & 6 & 40 & 60 & 70 \\
\hline & 24 & 80 & 80 & 80 \\
\hline
\end{tabular}

Table 3. Result of classification based on image using SVM with RBF kernel function.

\begin{tabular}{ccccc}
\hline Cultivar & Time & Low (\%) & Medium (\%) & High (\%) \\
\hline \multirow{2}{*}{ Fuji } & 6 & 80 & 100 & 100 \\
& 24 & 90 & 100 & 100 \\
Golden delicious & 6 & 100 & 100 & 100 \\
& 24 & 100 & 80 & 100 \\
Red delicious & 6 & 80 & 100 & 70 \\
& 24 & 90 & 100 \\
\hline
\end{tabular}

\section{CONCLUSIONS}

An effective hyperspectral imaging systems for detecting bruises in three apple cultivars was developed based on five wavelengths. The accuracy of the SVM and SVM with RBF kernel were 90.2 and $91.6 \%$. However, the SVM classification accuracy based on image analysis was decreased because of the misclassification of specular regions. Therefore, specular regions need to be removed by a polarizing filter or other image processing method. This result lays a foundation for development of a rapid detection system for bruised apples. Further research will pay attention to establishing a more precise waveband information, such as waveband resolution, and decreasing image acquisition time 


\section{REFERENCES}

[1] Keresztes, J. C., Goodarzi, M. and Saeys, W., "Real-time pixel based early apple bruise detection using short wave infrared hyperspectral imaging in combination with calibration and glare correction techniques," Food Control 66, 215-226 (2016).

[2] Xing, J., Bravo, C., Jancsók, P. T., Ramon, H. and De Baerdemaeker, J., "Detecting Bruises on 'Golden Delicious' Apples using Hyperspectral Imaging with Multiple Wavebands,” Biosyst. Eng. 90(1), 27-36 (2005).

[3] Che, W., Sun, L., Zhang, Q., Tan, W., Ye, D., Zhang, D. and Liu, Y., "Pixel based bruise region extraction of apple using Vis-NIR hyperspectral imaging," Comput. Electron. Agric. 146(July 2017), 12-21 (2018).

[4] Zhang, M. and Li, G., "Visual detection of apple bruises using AdaBoost algorithm and hyperspectral imaging," Int. J. Food Prop. 21(1), 1598-1607 (2018).

[5] Zhu, Q., Guan, J., Huang, M., Lu, R. and Mendoza, F., "Predicting bruise susceptibility of 'Golden Delicious' apples using hyperspectral scattering technique,” Postharvest Biol. Technol. 114, 86-94 (2016).

[6] M. S. Kim, Y. R. Chen and P. M. Mehl., "HYPERSPECTRAL REFLECTANCE AND FLUORESCENCE IMAGING SYSTEM FOR FOOD QUALITY AND SAFETY,” Trans. ASAE 44(3), 721-729 (2001).

[7] Qin, J., Chao, K., Kim, M. S., Lu, R. and Burks, T. F., "Hyperspectral and multispectral imaging for evaluating food safety and quality," J. Food Eng. 118(2), 157-171 (2013).

[8] Huang, W., Li, J., Wang, Q. and Chen, L., "Development of a multispectral imaging system for online detection of bruises on apples," J. Food Eng. 146, 62-71 (2015).

[9] ElMasry, G., Wang, N., Vigneault, C., Qiao, J. and ElSayed, A., "Early detection of apple bruises on different background colors using hyperspectral imaging," LWT - Food Sci. Technol. 41(2), 337-345 (2008). 